\title{
A CLASS OF RINGS WHICH ARE ALGEBRAIC OVER THE INTEGERS
}

\author{
DOUGLAS F. RALL \\ Department of Mathematics \\ Furman University \\ Greenville, South Carolina 29613 \\ U.S.A. \\ (Received June 23, 1978 and in Revised form October 30, 1978)
}

ABSTRACT. A well-known theorem of $\mathrm{N}$. Jacobson states that any periodic associative ring is commutative. Several authors (most notably Kaplansky and Herstein) generalized the "periodic polynomial" condition and were still able to conclude that the rings under consideration were commutative. (See [3]) In this paper we develop a structure theory for a class of rings which properly contains the periodic rings. In particular, an associative ring $R$ is sald to be a quasi-anti-integral (QAI) ring if for every $a \neq 0$ in $\mathrm{R}$ there exist a positive integer $k$ and integers $n_{1}, n_{2}, \ldots, n_{k}$ (all depending on $a$ ), so that $0 \neq n_{1} a=n_{2} a^{2}+$. $+n_{k} a^{k}$. In the main theorems of this paper, we show that any QAI-ring is a subdirect sum of prime QAI-rings, which in turn are shown to be left and right orders in division algebras which are algebraic over their prime fields. KEY WORDS AND PHRASES. Anti-integral, Quasi-anti-integral, Periodic, Prime. 1980 MATHEMATICS SUBJECT CLASSIFICATION CODES. Primary 16 A48 Secondary 16A12, 16A18, 16A40. 
1. INTRODUCTION.

In this paper we give a characterization of an associative ring which satisfies an algebraic condition over the integers. This condition can be viewed as a natural generalization of one studied by Osborn for a power-associative ring (1.e. a ring in which the subring generated by each element is associative). As in [5] $R$ will be called periodic if for every element a of $R$, there exists an integer $n=n(a)$ greater than 1 , such that $a^{n}=a$. In $[5, p .321]$ Osborn proves the following theorem which relates the periodic property to a more general kind of algebraic condition.

THEOREM 1. The following are equivalent for a power-associative ring R: 1. $R$ is a periodic ring.

2. Every element $a$ of $R$ is anti-integral over the ring of integers. That is, there are integers $k, n_{2}, n_{3}, \ldots ., n_{k}$ depending on a so that $n_{k} a^{k}+n_{k-1} a^{k-1}+\ldots+n_{2} a^{2}+a=0$.

3. $R$ is a discrete direct sum of 1deals each of which is an algebraic algebra over a periodic prime field and contains no nonzero nilpotent elements.

It follows that if $R$ is a periodic ring, then $R=T(R)$, where $T(R)=\{x \in R \mid n x=0$ for some integer $n \neq 0\}$ is the torsion 1deal of $R$. In this paper we consider a class of associative rings, which are not necessarily equal to their torsion ideals, but which satisfy a generalized anti-integral relation. In particular, if $\mathrm{F}$ is a commutative, associative ring with 1 , and $R$ is a power-associative F-algebra, then a nonzero element a of $R$ is said to be quasi-ant1 integral (QAI) over $F$ if there are 'scalars' $n_{1}, n_{2}, \ldots ., n_{k}$ In $F$ (depending on $a$ ) such that $0 \neq n_{1} a=n_{2} a^{2}+\ldots+n_{k} a^{k}$. R is a QAIalgebra over $F$ if every nonzero element of $R$ is QAI over $F$.

After developing some of the basic properties of QAI-algebras over $Z$, the ring of integers, we show that a prime associative ring which is QAI over $Z$ is 
a left and right order in a division algebra which is algebraic over its prime field, and conversely. We then show that an arbitrary associative QAI-algebra over the integers is a subdirect sum of prime QAI-rings. We present an example to show that not every such subdirect sum yields a QAI-ring.

\section{PROPERTIES OF QAI-ALGEBRAS.}

For the remainder of this paper, unless specifically stated otherwise, the term ring will mean associative ring without any assumption being made about the existence of a unity element. If $\mathrm{R}$ is a ring which is a QAI-algebra over

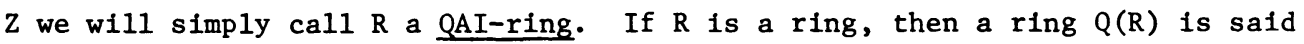
to be a left quotient ring for $R$ and $R$ is called a left order in $Q(R)$ provided (1) there is a monomorphism $h$ of $R$ into $Q(R)$ such that (ii) if $x$ is a regular element (i.e. one which is neither a left nor a right zero divisor) in $R$, then $h(x)$ is invertible in $Q(R)$, and (iii) $Q(R)$ consists of all elements of the form $h(a)^{-1} h(b)$ for $a, b$ in $R$ with a regular in $R$. Right quotient ring and right order are defined in a similar manner. If $R$ has a left quotient ring $Q(R)$, we will often assume that $R$ is a subring of $Q(R)$ and so dispense with the monomorphism $h$.

We state the following well-known results for future reference. See $[5],[3]$ for their proofs.

LEMMA 2. If $R$ is a left order in $Q(R)$ and $x_{1}, x_{2}, \ldots . ., x_{n}$ are arbitrary elements of $Q(R)$, then there are elements $a, b_{1}, b_{2}, \ldots . ., b_{n}$ in $R$, with a regular in $R$, such that $x_{1}=a^{-1} b_{1}(i=1,2, \cdots, n)$.

LEMMA 3. Suppose that $R$ is an F-algebra, where $F$ is a commutative and associative ring with 1 and with a left quotlent ring $Q(F)$. Then every element $x$ in $Q(F) \theta_{F} R$ can be written as $x=c^{-1} \theta$ for $c$ regular in $F$ and some $r$ in $R$.

The following lemma is well-known in its more general form, but we state it here and use it only for associative rings considered as algebras over $Z$. 
The proof in the general case may be found in [5, p. 189$]$.

LEMMA 4. Suppose $R$ is an associative algebra over the ring of integers $Z$, and that $T(R)=(0)$. Then the map $f$ from $R$ into $Q \theta_{Z} R$ given by $f(r)=1 \otimes r$ is a ring monomorphism. ( $Q$ is the field of rationals)

We observe that if $\mathrm{A}$ is a prime periodic ring of characteristic $\mathrm{p} \neq 0$, then $A$ is a periodic fleld and so $A$ is a left order and in fact $A=Q(A)$. In fact, the class of periodic fields is precisely the class of prime periodic rings which are left and right orders in fields of nonzero characteristic which are algebraic over their prime fields.

It follows from Theorem 1 that any periodic ring is a QAI-ring. In the characteristic zero case we will show that QAI rings play a role similar to that of periodic rings in characteristic $p$.

LEMMA 5. Let $D$ be any division algebra of characteristic zero which is algebraic over $Q$ and suppose $R$ is a subring of $D$. $R$ is a QAI-ring.

PROOF. Let a be a nonzero element of $R$. Since $D$ is Q-algebraic, there is a polynomial $\mathrm{p}(\mathrm{x})=\mathrm{r}_{\mathrm{n}} \mathrm{x}^{\mathrm{n}}+\ldots+\mathrm{r}_{2} \mathrm{x}^{2}+\mathrm{r}_{1} \mathrm{x}+\mathrm{r}_{0}$ in $Q[\mathrm{x}]$ such that $p(a)=0$. Assume such a polynomial of minimal degree has been chosen. Since a is not a zero divisor we may assume $r_{0} \neq 0$. But then $0 \neq r_{0} a=-\left(r_{n} a^{n+1}+\ldots+r_{2} a^{3}+r_{1} a^{2}\right)$. Suppose $r_{1}=c_{1} d_{1}^{-1}$ and let $d$ be the least common multiple of $d_{0}, d_{1}, \ldots, d_{n}$. Then $d_{1}$ is an integer for $1=0,1, \ldots, n$ and $0 \neq d r_{0} a=-d r a^{n+1}-\ldots-d_{2} a^{3}-d_{1} a^{2}$ so a is QAI, and $R$ is a QAI-ring.

The class of QAI-rings, although containing the periodic rings, can now be seen to be quite different from the class of periodic rings. The ring of integral quaternions is a noncommutative QAI-ring (compare with [2, Thm. 11]). A periodic ring is Jacobson semisimple since every element generates an idempotent, while the subring of $Q$ consisting of all quotients of the form 
$2 \mathrm{r} / 2 \mathrm{~s}+1,(2 \mathrm{r}, 2 \mathrm{~s}+1)=1$, is a Jacobson radical ring which is also QAI by Lemma 5 .

THEOREM 6. Let $R$ be an assoclative QAI-ring with torsion ideal $T(R)$. Then

(a) $R$ has no nonzero nilpotent elements, and thus every Idempotent of $R$ is contained in the center of $R, Z(R)$.

(b) $T(R)$ is a QAI-ring in its own right with the following decompositions:

(1) $T(R)$ is a periodic ring which is a direct sum of algebraic $\mathrm{Z}_{\mathrm{p}}$-algebras each of which has no nilpotent elements.

(11) $T(R)$ is a periodic ring which is a subdirect sum of primitive periodic rings (1.e. a subdirect sum of perfodic fields).

(c) If $S$ is any subring of $R$, then $S$ is a QAI-ring.

(d) Any homomorphic image of $R$ which is torsion-free is a QAI-ring.

PROOF. (a) It suffices to prove that $R$ has no nonzero elements which square to 0 . Let $\mathbf{r} \neq 0$ be an element of $R$. Then there are integers $n, k, a_{2}, a_{3}, \ldots, a_{k}$ such that $0 \neq n r=a_{2} r^{2}+a_{3} r^{3}+\ldots+a_{k} r^{k}$. Then clearly, $r^{2}=0$ implies $r^{2+1}=0$ for all positive integers 1 and this yields, $a_{2} r^{2}+a_{3} r^{3}+\ldots+a_{k} r^{k}=0$ a contradiction. Thus $r^{2} \neq 0$ and hence $R$ has no nonzero nilpotent elements. If $e^{2}=e$ and $x$ is an arbitrary element of $R$, then $(e x-e x e)^{2}=0=(x e-e x e)^{2}$. Thus ex $=$ exe $=x e$ and so $e$ is in $Z(R)$.

(b) $T(R)$ is an ideal of $R$ and it is clear that the property of being QAI is inherited by subrings, so $T(R)$ is itself a QAI-ring. 
(i) Let $R_{p}=\left\{x\right.$ in $R / p^{m} x=0$ for some positive integer $\left.m\right\}$, for each prime number $p . R_{p}$ is a subset of $T(R)$ and is in fact an ideal of $R$ and hence of $T(R)$. $T(R)$ is actually a discrete direct sum of the collection of ideals $\left\{R_{p}\right\}, p$ a prime. Let $x$ be an arbitrary element of $R_{p}$. There exists a positive integer $m$ such that $p^{m} x=0$. This implies $(p x)^{m}=p^{m} x^{m}=\left(p^{m} x\right) x^{m-1}=0$ so $p x$ is a nilpotent element of R. By (a), $p x=0$ and hence $R_{p}$ is an algebra over $z_{p}$. Also $0 \neq n x=a_{2} x^{2}+a_{3} x^{3}+\ldots+a_{t} x^{t}$ where $n, a_{2}, \ldots, a_{t}$ are integers which may be considered as coefficients from $z_{p}$ since $n x \neq 0$. Therefore, each $R_{p}$ is an algebraic $z_{p}$-algebra with no nonzero nilpotent elements and so a periodic ring by Theorem 1.

(11) By (1) $T(R)$ is a periodic ring and by $[2, T h m .11] T(R)$ is commutative. If a is an element of $T(R)$, then for some positive integer $m$ greater than $1, a^{m}=a$ which implies $e=a^{m-1}$ is an idempotent. But the Jacobson radical $J(T(R))$ contains no idempotents which yields a is not an element of $J(T(R))$. Thus $J(T(R))=(0)$ and $T(R)$ is a subdirect sum of primitive rings which must also be commutative and periodic, since they are homomorphic images of $T(R)$. Therefore $T(R)$ is a subdirect sum of periodic fields.

(c) This is clear since the QAI-property is an element-wise condition.

(d) If $I$ is an ideal of $R$ for which $R / I$ is torsion-free, then $R / I$ is

a QAI-ring follows from the fact that

$0 \neq n a=n_{2} a^{2}+n_{3} a^{3}+\ldots+n_{k} a^{k}$ implies

$\overline{0} \neq \overline{n a}=n_{2} \bar{a}^{2}+n_{3} \bar{a}^{-3}+\ldots+n_{k} \bar{a}^{-k}$ if $\bar{a}=a+I \neq \overline{0}$ in $R / I$. 
LEMMA 7. If $T(R)=0$ then $R$ is a QAI-ring if and only if every element of $R$ satisfies some polynomial with integer coefficients and has no nonzero nilpotent elements.

PROOF. The necessity follows directly from Theorem 6. Conversely, let w be a nonzero element of $R$. Then there is some polynomial $p(x)=a_{t} x^{t}+\ldots+$ $a_{2} x^{2}+a_{1} x$ with $a_{1}, a_{2}, \ldots, a_{t}$ integers so that $p(w)=0$. If $a_{1} \neq 0$ then $a_{1} w \neq 0$ and we are finished. Otherwise, let $k$ be minimal so that $a_{k} w 0$ and set $z=a_{t} w^{t-(k-1)}+\ldots+a_{k+1} w^{2}+a_{k} w$. If $z=0$, is QAI. Otherwise, $z \neq 0$ but $z^{k+1}=0$, a contradiction. Therefore $R$ is a QAI-ring.

It will sometimes be convenfent to use the obviously equivalent form of the definition: $a$ in $R$ is QAI if and only if there exists a polynomial $p(x)=a_{1} x+a_{2} x^{2}+\ldots+a_{k} x^{k}$ with integer coefficients so that $p(a)=0$ and $a_{1} a \neq 0 . \quad(p(x)$ will be called an integral polynomial.)

THEOREM 8. Suppose $R$ and $S$ are both power-associative QAI-rings. Then so is their direct sum $R \oplus S$.

PROOF. Let $(r, s)$ be an element of $R \oplus S$. We may assume that both $r$ and $s$ are nonzero for otherwise $(r, s)$ is QAI trivially. The argument will be divided into four cases determined by the additive orders of $r$ and $s$, which will be denoted by $o(r)$ and $o(s)$, respectively.

Case 1. $o(r)=n, o(s)=m$; $n$, m both finite. Then $r \varepsilon T(R)$ and $s \varepsilon T(S)$. By Theorem $6 \mathrm{~T}(\mathrm{R})$ and $T(S)$ are both periodic rings, and by the proof of Theorem $13.2[5]$ so is $T(R) \oplus T(S)$. But $T(R)+T(S)=T(R \bullet S)$. Thus $(r, s) \in T(R \oplus S)$, a periodic ring and hence $(r, s)$ is QAI.

Case 2. $o(r)=n$, finite and $o(x)=\infty$. Since $s$ is QAI there exists an integral polynomial $p(x)=a_{1} x+a_{2} x^{2}+\ldots+a_{k} x^{k}$ with $p(s)=0$ and $a_{1} s \neq 0$. Let $f(x)=n p(x)=\left(n a_{1}\right) x+\left(n a_{2}\right) x^{2}+\ldots+\left(n a_{k}\right) x^{k} \cdot f(x)$ is an integral polynomial and $\mathrm{na}_{1} \mathrm{~s} \neq 0$ since $\mathrm{s}$ has infinite additive order. Thus 


$$
\begin{aligned}
& n a_{1}(r, s)=\left(n a_{1} r, n a_{1} s\right)=\left(0, n a_{1} s\right) \neq(0,0) \text { in } R \oplus s \text {, yet } \\
& f((r, s))=n_{1}(r, s)+n a_{2}(r, s)^{2}+\ldots+n_{k}(r, s)^{k} \\
& =\left(n a_{1} r, n a_{1} s\right)+\left(n a_{2} r^{2}, n a_{2} s^{2}\right)+\ldots+\left(n a_{k} r^{k}, n a_{k} s^{k}\right) \\
& =(f(r), f(s))=(n p(r), n p(s))=(0,0)
\end{aligned}
$$

Therefore, $(r, s)$ is QAI.

Case 3. $o(r)=\infty, o(s)=n$, finite. Same as Case 2 - interchange the roles of $r$ and $s$.

Case 4. $o(r)=\infty, o(s)=\infty$. Since $R$ is a QAI-ring, there is an integral polynomial $p(x)=a_{1} x+a_{2} x^{2}+\ldots+a_{k} x^{k}$ with $a_{1} r \neq 0$ and $p(r)=0$. Now, $p((r, s))=a_{1}(r, s)+a_{2}(r, s)^{2}+\ldots+a_{k}(r, s)^{k}$

$$
\begin{aligned}
& =\left(a_{1} r, a_{1} s\right)+\left(a_{2} r^{2}, a_{2} s^{2}\right)+\ldots+\left(a_{k} r^{k}, a_{k} s^{k}\right) \\
& =(p(r), p(s))=(0, p(s)) .
\end{aligned}
$$

If $p(s)=0$ in $s$, then $p((r, s))=(0,0)$ and $a_{1}(r, s) \neq(0,0)$ in $R \oplus S$ so $(r, s)$ is QAI. Otherwise, $\mathrm{p}(s) \neq 0$ in $\mathrm{s}$. But $\mathrm{S}$ is a QAI-ring so there is an integral polynomial $h(x)=b_{1} x+b_{2} x^{2}+\ldots+b_{n} x^{n}$ with $b_{1} p(s) \neq 0$ and $h(p(s))=0$ in $s$. Define $g(x)$ to be the composition of the two polynomials $p(x)$ and $h(x)$. That is, set

$$
\begin{aligned}
g(x)=h(p(x)) & =b_{1}\left(a_{1} x+a_{2} x^{2}+\ldots+a_{k} x^{k}\right) \\
& +b_{2}\left(a_{1} x+a_{2} x^{2}+\ldots+a_{k} x^{k}\right)^{2}+\ldots \\
& +b_{n}\left(a_{1} x+a_{2} x^{2}+\ldots+a_{k} x^{k}\right)^{n}
\end{aligned}
$$

Upon inspection of this polynomial one sees that the coefficient of the linear term is $b_{1} a_{1} \neq 0$ and that $g(x)$ is clearly an integral polynomial. Since $o(r)=\infty=o(s)$, we get $b_{1} a_{1}(r, s)=\left(b_{1} a_{1} r, b_{1} a_{1} s\right) \neq(0,0)$ the zero element of $R \oplus S$. However, 
$g((r, s))=h(p((r, s)))=h((0, p(s)))=(0, h(p(s)))=(0,0)$, and $(r, s)$ is

QAI. Since these four cases are exhaustive, the theorem is proved.

COROLLARY 9. Suppose $R_{1}, R_{2}, \ldots, R_{n}$ are QAI-rings. Then their direct sum $R_{1} \oplus R_{2} \oplus \ldots \oplus R_{n}$ is also a QAI-ring.

PROOF. This can be proved easily by induction.

COROLLARY 10. Let $\left\{R_{t}\right\}_{t} \in A$ be any collection of QAI-rings, and suppose $R=\sum_{t}^{\oplus A} R_{t}$ is their weak direct sum. Then $R$ is a QAI-ring.

PROOF. Let $a$ be any nonzero element of $R$. Then all but a finfte number of the components of a are zero; i.e. If $a=\left(a_{t}\right)_{t} \varepsilon A$ then there exist $a$ finite number of elements $t_{1}, t_{2}, \ldots, t_{k}$ in A such that $a_{s}=0$ if $s$ is not a member of $\left\{t_{1}, t_{2}, \ldots, t_{k}\right\}$. Then a can be considered to be an element of $R_{t_{1}} \oplus R_{t_{2}} \oplus \ldots \oplus R_{t_{k}}$ which is a QAI-ring. Thus a is QAI and $R=\underset{t \in A}{\oplus \sum_{t}} R_{t}$ is a QAI-ring.

As can be seen from Theorem 6 and Corollary 10, the class of QAI-rings is closed under taking subrings, direct sums and torsion-free homomorphic images. However, $Z / \mathrm{nZ}$ is a homomorphic image of $Z$ which is not a QAI-ring whenever $n$ is not square-free. To see that the class of QAI-rings is not closed under complete direct products consider the following example.

EXAMPLE. Let $\mathrm{w}_{1}=\mathrm{z}$, the ring of integers for $1=1,2,3, \ldots$, and set $R=\prod_{1=1}^{\infty} W_{1}$. Let $x \in R$ be that element $x=\left(x_{1}\right) \quad$ such that $x_{1}=1$. Suppose $x$ is QAI. That is, suppose there exist $n, a_{2}, a_{3}, \ldots, a_{k}$ integers so that $0 \neq n \mathrm{nx}=\mathrm{a}_{2} \mathrm{x}^{2}+\mathrm{a}_{3} \mathrm{x}^{3}+\ldots+\mathrm{a}_{\mathrm{k}} \mathrm{x}^{k}$. Since two elements of $\mathrm{R}$ are equal if and only if they are equal in each component, this equation yields the following 
infinite system of equations:

$$
\begin{aligned}
& n=a_{2}+a_{3}+\ldots+a_{k} \\
& 2 n=4 a_{2}+8 a_{3}+\ldots+2^{k} a_{k}-\text { which implies that } 2 \text { divides } n \\
& 3 n=9 a_{2}+27 a_{3}+\ldots+3^{k} a_{k}-\text { which implies that } 3 \text { divides } n \\
& \text {. } \\
& m n=m^{2} a_{2}+m^{3} a_{3}+\ldots+m^{k} a_{k}-\text { which implies that m divides } n
\end{aligned}
$$

This is clearly a contradiction since $n$ can have only a finite number of divisors. Therefore $R$ is not a QAI-ring.

\section{THE PRIME CASE}

Let $R$ be an arbitrary $Q A I-r i n g$, and $P(R)$ be the prime radical of $R$. $P(R)$ is the intersection of all the prime ideals of $R$ and is also a nil ideal of $R$, hence equals $(0)$, since $R$ has no nilpotent elements and thus certainly no nfl 1deals. This fact, together with examples of QAI-rings which are Jacobson radical rings, led us to investigate QAI-rings via the prime-semi-prime route instead of the primitive-semisimple one.

LEMMA 11. If $R$ is a prime associative ring then $Q \theta_{Z} R$ is also prime. PROOF. If $R$ is prime, then it has a characteristic $p$. If $p \neq 0$, then $Q \theta_{Z} R=(0)$ since $\mathrm{m} / \mathrm{n} \otimes \mathrm{r}=\mathrm{mp} / \mathrm{np} \otimes \mathrm{r}=\mathrm{m} / \mathrm{np} \otimes \mathrm{pr}=\mathrm{m} / \mathrm{np} \otimes 0=0$.

If $p=0$, then by Lemma $4, R$ is embedded in $Q \theta_{Z} R$ via $f: r \longrightarrow 10 \mathrm{r}$. Suppose there exist elements $x, y$ in $Q \theta_{Z} R$ such that $x\left(Q \theta_{z} R\right) y=(0)$. By Lemma $3, x=s^{-1} \otimes r_{1}, y=t^{-1} \otimes r_{2}$ for some $s, t \varepsilon z$ and $r_{1}, r_{2} \varepsilon R$. Then $0=\left(s^{-1} \otimes r_{1}\right)(Q \otimes R)\left(t^{-1} \otimes r_{2}\right)=\left(s^{-1} Q t^{-1}\right) \otimes\left(r_{1} R r_{2}\right)$. In particular 
$0=\left(s^{-1}(s t) t^{-1}\right) \theta\left(r_{1} R r_{2}\right)=10\left(r_{1} R r_{2}\right)$. But $f: R \longrightarrow Q Q_{2} R$ Is one-to-one and so $r_{1} R r_{2}=0$. But $R$ is prime and so $r_{1}=0$ or $r_{2}=0$.

Therefore, $x\left(Q_{z} R\right) y=(0)$ implies $x=0$ or $y=0$ and $Q \theta_{z} R$ is prime.

LEMMA 12. Suppose $R$ is a prime ring with no nonzero nilpotent elements.

Then $R$ has no nonzero, zero-divisors.

PROOF. Suppose $x y=0$. By hypothesis $R$ has no nonzero nilpotent elements. Now $(y R x)^{2}=(y R x)(y R x)=y R(x y) R x=0$ and thus $y R x=0$. The primeness of $R$ now forces $x=0$ or $y=0$. Therefore $x y=0$ implies $x=0$ or $y=0$.

LEMMA 13. Suppose $R$ is a prime QAI-ring of characteristic zero. Then $S=Q \theta_{Z} R$ is also a QAI-ring.

PROOF. Let $x \in Q 0_{Z} R$. By Lemma 3, there is an integer $c$ and an element $r$ in $R$ such that $x=c^{-1}$ or. $S$ is a Q-algebra, so we may rewrite $x$ as $x=c^{-1}\left(\begin{array}{lll}1 & \bullet & r\end{array}\right)$.

$R$ is a QAI-ring and $r \varepsilon R$ so there are integers $n, n_{2}, n_{3}, \ldots, n_{k}$ such that $0 \neq n r=n_{2} r^{2}+n_{3} r^{3}+\ldots+n_{k} r^{k}$. Since $S$ is a Q-algebra, it is torsionfree so

$$
\begin{aligned}
0 \neq n(10 r) & =n \theta r=10 n r=10\left(n_{2} r^{2}+n_{3} r^{3}+\ldots+n_{k} r^{k}\right) \\
& =\left(10 n_{2} r^{2}\right)+\left(10 n_{3} r^{3}\right)+\ldots+\left(10 n_{k} r^{k}\right) \\
& =n_{2}(10 r)^{2}+n_{3}(10 r)^{3}+\ldots+n_{k}(10 r)^{k} \text {. Now } \\
0 \neq n x & =n\left(c^{-1} \text { Or }\right)=c^{-1}(n \theta r)=c^{-1}\left(n_{2}(10 r)^{2}+n_{3}(10 r)^{3}+\ldots+n_{k}(10 r)^{k}\right) \\
& =c^{-1} n_{2}(10 r)^{2}+c^{-1} n_{3}(10 r)^{3}+\ldots+c^{-1} n_{k}(10 r)^{k} \\
& =n_{2} c\left(c^{-1} \text { Or }\right)^{2}+n_{3} c^{2}\left(c^{-1} \text { or }\right)^{3}+\ldots+n_{k} c^{k-1}\left(c^{-1} \text { Or }\right)^{k} \text {, since s }
\end{aligned}
$$

is a Q-algebra. But if $a_{1}=n_{1} c^{1-1}$ for $1=2,3, \ldots, k$ then we have shown that $0 \neq n x=a_{2} x^{2}+a_{3} x^{3}+\ldots+a_{k} x^{k}$ with $a_{1} \varepsilon z$. Therefore since $x \in S$ was arbitrary, $S$ is a QAI-ring which is also a Q-algebra. 
COROLLARY 14. If $R$ is a prime QAI-ring of characteristic zero, then $R$ can be embedded as a subring in a Q-algebra $S$ which is a prime QAI-ring.

PROOF. Let $S=Q \theta_{Z} R$. By Lemma 11 and Lemma 13, $S$ is a prime QAI-ring and is clearly a Q-algebra. By Lemma $4 \mathrm{R}$ is embedded inside $\mathrm{S}$ by $\mathrm{r} \longrightarrow 10 \mathrm{r}$.

If we require, in addition to the hypothesis of the last corollary, that our ring $R$ have a unity element 1 , then we can also conclude that $Q \theta_{Z} R$ is a division algebra. In particular, let $S$ be a Q-algebra which is a prime QAI-ring with 1 and let $x$ be a nonzero element of $S$. We wish to show that $x$ is invertible. There exist integers $m, a_{2}, a_{3}, \ldots, a_{t}$ so that $0 \neq m x=a_{2} x^{2}+a_{3} x^{3}+\ldots+a_{t} x^{t}$. Then $0=m x-\left(a_{2} x^{2}+\ldots+a_{t} x^{t}\right)=x\left(m \cdot 1-\left(a_{2} x+\ldots+a_{t} x^{t-1}\right)\right)$. But by Lemma 12 , $R$ has no zero-divisors and then we get $m \cdot 1-\left(a_{2} x+a_{3} x^{2}+\ldots+a_{t} x^{t-1}\right)=0$. Thus $m \cdot 1=a_{2} x+a_{3} x^{2}+\ldots+a_{t} x^{t-1}=\left(a_{2} \cdot 1+a_{3} x+\ldots+a_{t} x^{t-2}\right) \cdot x$. So $x$ is invertible, $x^{-1}=m^{-1}\left(a_{2} \cdot 1+a_{3} x+\ldots+a_{t} x^{t-2}\right)$, and we have proven the following.

THEOREM 15. If $R$ is a prime Q-algebra with 1 which is also a QAI-ring then $R$ is a division algebra.

If one assumes that $R$ is a prime QAI-ring with 1 , then the resulting Q-algebra $Q{ }_{Z} R$ satisfies the hypothesis of Theorem 15. The following theorem shows that we need not assume the existence of 1 .

THEOREM 16. If $R$ is a prime Q-algebra which is also a QAI-ring, then $R$ is a division algebra, algebraic over Q.

PROOF. Let a be a nonzero element of $R$. There exist positive integers $n, k$ and integers $n_{2}, n_{3}, \ldots, n_{k}$ such that $0 \neq n a=n_{2} a^{2}+\ldots+n_{k} a^{k}$. We may assume that $n_{k} \neq 0$ - for otherwise the polynomial is of smaller degree and at least one of the $n_{1} \neq 0$. Since $R$ is a Q-algebra, 
$a^{k}=n_{k}^{-1}\left(n a-\left(n_{2} a^{2}+\ldots+n_{k-1} a^{k-1}\right)\right)$. That 1s, A, the Q-subalgebra of $R$ generated by $a$ is finite dimensional.

Consider $a_{r}: A \longrightarrow A$, the right multiplication map given by $a_{r}(x)=x a$. $a_{r}$ is a Q-1inear transformation which is one-to-one since $R$, being a prime QAI-ring, has no nonzero zero-divisors. Now A is finite dimensional $80 a_{r}$ is also onto, and by a standard argument $a_{r}$ is invertible and hence $a$ is invertible in A. Similarly, every $b$ in $A$ which is nonzero 18 invertible, and since $A$ is a commutative subalgebra, it is a field. Let $e$ in $A$ be the 1dentity of $A$. $e^{2}=e$, and for any $y \neq 0$ in $R, e(e y-y)=0=(y e-y) e$. Since $R$ has no nonzero zero-divisors, ey $=y=y e$ and $e$ is an ident1ty for $R$. Thus every nonzero element of $R$ is invertible and $R$ is a division algebra. The fact that $R$ is algebraic over $Q$ follows from the definition of QAI-ring.

Having characterized prime Q-algebras which are QAI-rings as being division algebras which are algebraic over the rationals, we now wish to investigate the nature of the embedding of a prime, characteristic zero $Q A I-r i n g R$ into $Q \theta_{z} R$. It is interesting that $R$ turns out to be a left and right order in $S=Q_{Z} R$. To demonstrate this, it is convenient to know the inverse of an arbitrary nonzero element $c^{-1}$ or in $s$.

LEMMA 17. Suppose $e=t^{-1} \theta s$ is the 1dentity in $S$ and $c^{-1}$ Or 18 any nonzero element of $\mathrm{S}$. Then the multiplicative inverse of $c^{-1}$ or is given by $d^{-1}$ Ou where $\operatorname{tru}=\mathrm{cds}=$ tur.

PROOF. By Theorem 16, $\mathrm{c}^{-1}$ Or is invertible since $S$ is a division algebra. Suppose $\left(c^{-1} \theta r\right)^{-1}=d^{-1} \theta u$. Then $\left(c^{-1} \theta r\right)\left(d^{-1} \theta u\right)=t^{-1} \theta s=\left(d^{-1} \theta u\right)\left(c^{-1} \theta r\right)$, if and only if (cd $)^{-1}$ Oru $=t^{-1} \theta s=(d c)^{-1}$ Our. Since $S$ is a Q-algebra, upon

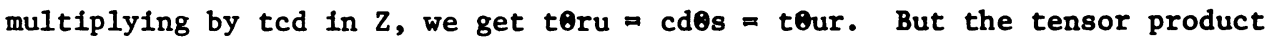
is taken over $\mathrm{Z}$, so $10 \mathrm{tru}=10 \mathrm{cds}=10 \mathrm{tur}$. Applying Lema 14 this is equivalent to tru $=c d s=$ tur. 
We now proceed to characterize prime QAI-rings of characteristic zero. THEOREM 18. Let $R$ be a prime QAI-ring of characteristic zero. Then $R$ is a left and right order in a rational division algebra which is algebraic over $Q$.

PROOF. By Corollary 14 and Theorem 16 the function $f: R \longrightarrow Q_{Z} R$ given by $f(r)=10 r$ is a ring monomorphism of $R$ into the Q-division algebra $S=Q \theta_{Z} R$ which is algebraic over the rational field. It remains then to show that $f$ actually embeds $R$ as a left and right order in $S$.

By definition, we must show:

(1) If $x$ is regular in $R$, then $f(x)$ is invertible in $S$; and

(ii) Every element $z$ in $S$ can be written as $z=f(x)^{-1} f(y)$ (and $z=f(u) f(v)^{-1}$ ) where $x, y, u, v$ are in $R$ with $x$ and $v$ regular. To establish (1), it is necessary only to observe that since $f$ is one-to-one and $S$ is a division algebra, $f(x)=10 x$ will be invertible for any $x \neq 0$ in $R$.

Let $e=t^{-1} \theta s$ be the identity for $S$. From $(10 \mathrm{~m})\left(t^{-1} \theta s\right)=10 \mathrm{~m}=\left(t^{-1} \theta \mathrm{s}\right)(10 \mathrm{~m})$ it follows that $t^{-1}$ Oms $=10 \mathrm{~m}=t^{-1}$ Osm which implies (multiplying by $t$ ) $10 \mathrm{~ms}=10 \mathrm{tm}=10 \mathrm{sm}$ and hence, because $\mathrm{f}$ is one-to-one, $\mathrm{ms}=\mathrm{tm}=\mathrm{sm}$.

Let $z$ be an arbitrary element of $S$, say $z=p^{-1}$ m where $p \neq 0$ is an integer and $m$ is in $R$. If $m=0$, then write $z=(10 y)^{-1}(100)$ for any $y \neq 0$ in $R$. So assume $m \neq 0$; then $\mathrm{pm} \neq 0$ since $R$ is prime of characteristic zero. Consider $10 \mathrm{pm} \neq 0$. By Lemma $17(10 \mathrm{pm})^{-1}=\mathrm{q}^{-1}$ Ow where $\mathrm{tpwm}=\mathrm{tpmw}=\mathrm{qs}$. Then $(10 \mathrm{pm})^{-1}\left(10 \mathrm{~m}^{2}\right)=\left(\mathrm{q}^{-1} \Theta \mathrm{w}\right)\left(10 \mathrm{~m}^{2}\right)=\mathrm{q}^{-1} \mathrm{wm}^{2}$. But $\mathrm{tpwm}=\mathrm{qs}$, and upon multiplying by $m$ in $R$, we get $\mathrm{tpwm}^{2}=q s m=s q m=t q m$. Thus $t\left(\mathrm{pwm}^{2}-\mathrm{qm}\right)=0$ and $R$ being torsion-free implies $\mathrm{pwm}^{2}=\mathrm{qm}$. It follows that $10 \mathrm{pwm} \mathrm{m}^{2}=\mathrm{f}\left(\mathrm{pwm}^{2}\right)=\mathrm{f}(\mathrm{qm})=10 \mathrm{qm}$. Since $S$ is a $Q$-algebra, if we multiply by $(\mathrm{pq})^{-1}$ then we see that $\mathrm{q}^{-1} \theta \mathrm{wm}^{2}=$ $\mathrm{p}^{-1} \mathrm{Om}=\mathrm{z}$. 
Therefore $z=p^{-1} \theta m=q^{-1} \theta w^{2}=\left(q^{-1} \theta w\right)\left(10 m^{2}\right)=(10 p m)^{-1}\left(10 m^{2}\right) . \quad s$ is then seen to be the collection of all elements of the form $(10 x)^{-1}(10 y)=$ $f(x)^{-1} f(y)$. Thus $R$ is a left order in S. The proof for ' $R$ is a right order In $S^{\prime}$ is similar to the one above.

We now turn our attention to the remaining case in the prime situation-that of prime QAI-rings of nonzero characteristic. Indeed it is here that we find the overlap with periodic rings.

THEOREM 19. Suppose $R$ is a prime QAI-ring of characteristic $p \neq 0$. Then $R$ is a periodic field.

PROOF. Because $R=T(R)$, by Theorem $6 \quad R$ is a periodic ring and by Lemma $12, R$ has no nonzero zero-divisors. If $x \neq 0$ in $R$ then there exists a positive integer $n$ greater than 1 such that $x^{n}=x$. Then if $e=x^{n-1}, e^{2}=e \neq 0$. We claim that $e$ must be the identity element for $R$. If not, then there is $y$ in $R$ such that $e y \neq y$ (or ye $\neq y$ ). But then ey $-y \neq 0$ and yet $e(e y-y)=0$ (or ye $-y \neq 0$ and $(y e-y) e=0$ ). This yields a contradiction since $R$ has no nonzero zero-divisors and hence $e=x^{n-1}$ is an identity for $R$. For any $w$ in $R, w^{k}=w$ for some $k$ greater than 1 and as above $w^{k-1}$ is an idempotent. By the same argument as before $\mathrm{w}^{\mathrm{k}-1}=\mathrm{e}$. Thus every nonzero element of $\mathrm{R}$ is Invertible and by [2, Thm. 11] $R$ is commutative. Therefore a prime QAI-ring of characteristic $p \neq 0$ is a periodic field.

THEOREM 20. For a ring $R$ to be a prime QAI-ring it is necessary and sufficient that it be a left and right order in a division algebra which is algebraic over its prime field.

PROOF. The necessity follows directly from Theorem 18 (characteristic zero) and Theorem 19 (characteristic $p$ ). For sufficiency, let $R$ be a left and right order in a division algebra $D$, where $D$ is algebraic over its prime 
field F. Suppose $x \neq 0$ in $R$; then there exist a positive integer $n$ and $a_{0} \neq 0, a_{1}, \ldots, a_{n}$ In $F$ such that $a_{n} x^{n}+\ldots+a_{1} x+a_{0}=0$. Multiplying by $x$ gives $a_{n} x^{n+1}+\ldots+a_{1} x^{2}+a_{0} x=0$.

Case 1. If char $F=p \neq 0$ then the $a_{1}$ can be considered as integers (between 0 and $\mathrm{p}$ ).

Case 2. If char $F=0$, then $F=Q$ and $a_{1}=b_{1} c_{1}^{-1}, 1=0,1, \ldots, n$. Set $d$ equal to the least common multiple of $c_{0}, c_{1}, \ldots, c_{n}$. Then da ${ }_{1}$ is an integer for $1=0,1, \ldots, n$ and $\left(d a_{n}\right) x^{n+1}+\ldots+\left(d a_{1}\right) x^{2}+\left(d a_{0}\right) x=0$.

In either case $x$ is $Q A I$ so $R$ is a QAI-ring. Since a subring of a division ring is always prime $R$ is a prime QAI-ring.

In fact, we can drop the assumption that $R$ be an order in Theorem 20 .

COROLLARY 21. A ring $R$ is a prime QAI-ring if and only if $R$ is a subring of a division algebra which is algebraic over its prime field.

There are several instances where a QAI-ring $R$, being a special type of prime ring, must not only be a left order in a division ring but must itself be a division ring.

THEOREM 22. Suppose $R$ is a primitive QAI-ring. Then $R$ is a division ring which is algebraic over its prime field.

PROOF. A primitive ring is prime; so if $R$ has nonzero characteristic, it will be a periodic field by Theorem 19. We thus assume that $R$ is a primftive QAI-ring of characteristic zero. By the Density Theorem $R$ is isomorphic to a dense ring of linear transformations of a vector space $V$ over a division ring $D$. We claim that the D-dimension of $V$ is one. Suppose not; that 1s, suppose that $\left\{v_{1}, v_{2}\right\}$ is a D-linearly independent subset of $V$. Since $R$ is dense, there exist elements $x$ and $y$ in $R$ so that $y v_{1}=v_{2}, y v_{2}=0, x v_{1}=0, x v_{2}=v_{2}$. But $R$ is a QAI-ring so there exist integers $m, m_{2}, m_{3}, \ldots, m_{t}$ and $n, n_{2}, n_{3}, \ldots, n_{s}$ so that $0 \neq m x=m_{2} x^{2}+m_{3} x^{3}+\ldots+m_{t} x^{t}$ and $0 \neq n y=n_{2} y^{2}+n_{3} y^{3}+\ldots+n_{s} y^{s}$. 
Then mnxy $=m x\left(n_{2} y^{2}+\ldots+n_{s} y^{s}\right)=a y^{2}$, where $a=m x\left(n_{2}+n_{3} y+\ldots+n_{s} y^{s-2}\right)$. But $0=0 \cdot v_{1}=\left(\operatorname{mnxy}-a y^{2}\right) v_{1}=\operatorname{mnx}\left(y v_{1}\right)-a y\left(y v_{1}\right)=m n v_{2}$, which is a contradiction since $D$ has characteristic zero if $R$ does. Thus the dimension of $V$ over $D$ is one and so $R$ is isomorphic to $D=\operatorname{Hom}_{D}(V, V)$, and $R$ is a division ring. $R$ is algebraic over its prime field by the definition of QAI.

COROLLARY 23. Suppose $R$ is a QAI-ring which is an algebra over a fleld F. Then $R$ is a subdirect sum of division algebras over $F$.

PROOF. Since $R$ is a QAI-ring, $R$ is algebraic over $F$. Thus the Jacobson radical is a nil ideal of $R$ and hence $(0)$. $R$ is then a subdirect sum of primitive F-algebras, $\left\{D_{t}\right\}_{t \in A}$. Each $D_{t}$ is a homomorphic image of $R$ and has the same characteristic as $R$ so is a QAI-ring. The corollary follows from Theorem 22.

THEOREM 24. A simple QAI-ring must be a division ring.

PROOF. For each positive integer $m$, let $m R=\{m r \mid r \varepsilon R\}$. $m R$ is an ideal of $R$, and since $R$ is simple, either $m R=(0)$ or $m R=R$. If there is an $m$ for which $m R=(0)$, then $R$ is a prime $Q A I-r i n g$ of nonzero characteristic. By Theorem $19 \mathrm{R}$ is a periodic field. Otherwise, $m R=R$ for every positive integer m. In this case $R$ is a Q-algebra and so a division ring by Theorem 16 .

\section{THE GENERAL CASE.}

In our study of prime QAI-rings we considered two separate cases - one of characteristic zero and another of prime characteristic. If $R$ is any QAI-ring, then $R$ is semiprime; however, a semiprime ring may have characteristic zero and still contain elements of finite order. The dichotomy we will use in the general case will be the torsion $T(R)$ and the torsion-free $R / T(R)$ cases, then attempting to put these two extremes together to say something about $R$. As is often the case, it is the latter which is the most difficult. 
THEOREM 25. Let $R$ be a QAI-ring. The torsion 1deal, $T(R)$, is a subdirect sum of perlodic flelds and $R / T(R)$ is a subring of an algebra $S$ over the rationals $Q$, so that $S$ is a subdirect sum of Q-division algebras which are algebraic over $Q$.

PROOF. The conclusions about $T(R)$ are just Theorem 6 . Let $M=R / T(R)$, a torsion-free $\mathrm{QAI}-\mathrm{ring}$ by Theorem 6. Set $\mathrm{S}=\mathrm{Q}_{\mathrm{Z}} \mathrm{M}$. Since $\mathrm{M}$ is a $\mathrm{QAI-ring}$, so is $S$ by Lemma 13. If $\mathrm{f}: \mathrm{M} \longrightarrow \mathrm{S}$ is given by $\mathrm{f}(\mathrm{m})=10 \mathrm{~m}$ then $\mathrm{f}$ is a ring monomorphism since $M$ is torsion-free. Thus we may consider $M$ as a subring of a Q-algebra S which is itself a QAI-ring. We claim that $S$ is a subdirect sum of prime Q-algebras which are QAI-rings. S has no nonzero nilpotent elements and hence no nonzero nil 1deals. Suppose a is a nonzero element of $\mathrm{S}$, and set $J_{a}=\left\{I \mid I\right.$ is a Q-algebra ideal of $S$ and $I n\left\{a^{n} \mid n\right.$ is a positive integer $\left.\}=\phi\right\}$. (0) $\varepsilon \mathrm{J}_{\mathrm{a}}$ so $\mathrm{J}_{\mathrm{a}} \neq \phi$. By Zorn's Lemma pick $\mathrm{I}_{\mathrm{a}} \operatorname{maximal}$ in $\mathrm{J}_{\mathrm{a}}$. Then $\mathrm{I}_{\mathrm{a}}$ is a prime algebra ideal, and the intersection of the collection $\left\{I_{a} \mid a \varepsilon S\right\}$ of ideals is (0). Each $S / I_{a}$ is a Q-algebra which forces it to be torsion-free and hence a QAI-ring by Theorem 6. Thus $S$ is a subdirect sum of the collection $\left\{S / I_{a}\right\} a s$ of prime Q-algebras which are QAI-rings, and we are done by applying Theorem 16 . Suppose $R$ is a torsion-free QAI-ring. It follows from Theorem 25 that we may embed $R$ in a rational algebra which has a nice representation. Can we then say that $R$ also has a similar representation?

THEOREM 26. Suppose $R$ is a torsion-free QAI-ring. Then $R$ can be written as a subdirect sum of orders in Q-division algebras each of which is algebraic over $Q$.

PROOF. Since $Q$ is the quotient field of $Z$, the homomorphism $f: R \longrightarrow Q_{Z}^{R}$ given by $f(r)=10 \mathrm{r}$ is a ring embedding. By the proof of Theorem $25, Q Z_{2} \mathrm{is}$ a subdirect sum of a collection of Q-division algebras $\left\{D_{t}\right\}_{t} \varepsilon A^{*}$ For each $t$ in $A$, $D_{t}$ is algebraic over $Q$. Consider the following diagram: 


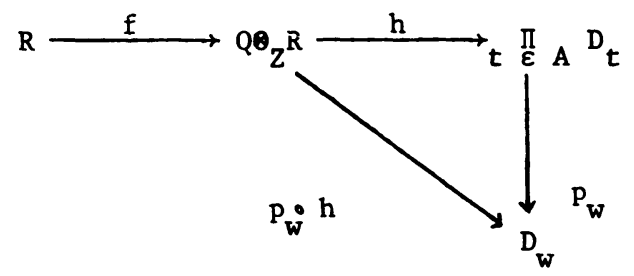

$h$ is the embedding of ${ }_{Z} R$ into the complete direct product, $t \prod_{\varepsilon}{ }_{A}{ }_{t}$ and $P_{w}$ is just the projection homomorphism onto the wth coordinate. Set $E_{w}=p_{w}(h(f(R)))$. $E_{w}$ is a subring of $D_{w}$ and a ring homomorphic image of $R$. In fact, $E_{w}$ is an order In $D_{w}$. For if $d$ is an element of $D_{w}$ and 1 is the unity of $D_{w}$, then there are elements $n^{-1} \theta e$ and $m^{-1} \theta r$ such that $p_{w}\left(h\left(n^{-1} \theta e\right)\right)=1$ and $p_{w}\left(h\left(m^{-1} \theta r\right)\right)=d$. Then $p_{w}(h(f(m e)))=m n \cdot 1$ and $p_{w}(h(f(n r)))=n m d$. Thus $d=(m n \cdot 1)^{-1}(m n d)=$ $\left(p_{w}(h(f(m e)))\right)^{-1}\left(p_{w}(h(f(n r)))\right)$. By the way $E_{t}$ is defined for each $t$ in $A$, it is clear that $R$ is a subdirect sum of $\left\{E_{t}\right\}_{t} \in A^{*}$. (The proof that $E_{w}$ is an order in $D_{w}$ is due to Professor F. Kosier.)

COROLLARY 27. If $R$ is a torsion-free QAI-ring, then $R$ is a subdirect sum of prime QAI-rings of characteristic zero.

PROOF. Using the notation of the proof of Theorem $26, R$ is a subdirect sum of $\left\{E_{t}\right\}_{t \in A^{*}} E_{t}$ is a subring (order) of a Q-division algebra and is a QAI-ring. Thus $E_{t}$ is prime of characteristic zero.

Reviewing what we have done up to this point in the general case, we see that by Theorem 25 and Theorem 26 if $R$ is either a torsion QAI-ring or a torsionfree QAI-ring, then $R$ is a subdirect sum of orders in division algebras, each being algebraic over its prime field. Our present goal is to get a similar decomposition for an arbitrary QAI-ring.

DEFINITION. If $R$ is a ring then a subset $M$ of $R$ is called a multiplicative system if

1) 0 is not in $M$, and

2) whenever $m$ and $n$ are elements of $M$, so is their product mn. 
$M$ is a maximal multiplicative system if it is not a proper subset of another multiplicative system.

Observe that if $R$ is a QAI-ring, then $R$ has no nonzero nilpotent elements; so if $r$ is a nonzero element of $R, N_{r}=\left\{r^{n} \mid n=1,2,3, \ldots\right\}$ is a multiplicative system. We state the following three lemmas without proof; see [1].

LEMMA 28. If $R$ is a $Q A I-r i n g$ and $a \neq 0$ in $R$, then there is a maximal multiplicative system containing a.

LEMMA 29. If $M$ is a maximal multiplicative system in a ring $R$ without nilpotent elements, then the set-theoretic complement $c(M)$ is an ideal.

LEMMA 30. Let $R$ be a ring without nilpotent elements and for each $x \neq 0$ in $R$ let $M_{x}$ be a maximal multiplicative system of $R$ containing $x$ with complement $c\left(M_{x}\right)$. Then $R$ is a subdirect sum of the integral domains $\left\{R / c\left(M_{x}\right)\right\}_{x} \neq 0 \varepsilon R^{\circ}$

As we observed earlier, all homomorphic images of a QAI-ring need not be QAI-rings. In Theorem 6 we showed that if $I$ is an ideal of $R$ such that $R / I$ is torsion-free then $R / I$ is a QAI-ring. However, in the decomposition of $R$, we can make use of the following:

LEMMA 31. Let $R$ be a QAI-ring and $M$ a maximal multiplicative system in $R$. Then $R / c(M)$ is a QAI-ring.

PROOF. For notational convenience we denote $R / C(M)$ by $A$ and let $\bar{x}$ represent the image of $x$ under the natural homomorphism $f: R \longrightarrow A$. From Lemma 30 we see that $\mathrm{A}$ is an integral domain. If $\mathrm{A}$ has characteristic zero, then we are finished since it will then be torsion-free. Thus we may assume A has characteristic a prime $\mathrm{p} \neq 0$. We make two observations:

(i) Since A has no zero divisors, it suffices to show that for a in $R$ with $\bar{a} \neq \overline{0}, \bar{a}$ satisfies some relation of the form $n_{k} x^{k}+n_{k-1} x^{k-1}+\ldots+n_{2} x^{2}+n_{1} x=0$ with at least one--any one--of the integers $n_{1}$ not divisible by $p$. 
(i1) If a is a nonzero element of $R$ of finite additive order $m$, then $\overline{m a}=m f(a)=f(m a)=f(0)=\overline{0}$. Thus $f(a)=\bar{a}$ has finite additive order $k$ and $k$ divides $m$. Therefore, since the characteristic of $A$ is $p$, if $a$ in $R$ is of order $q$ where $q$ is a prime, $q \neq p$, then $\bar{a}=f(a)=\overline{0}$ in $A$.

$T(R)=\oplus \Sigma R_{p}$ where $R_{p}=\left\{x \in R \mid p^{k} x=0\right.$ for some $\left.k\right\}$

$$
=\{\mathrm{x} \varepsilon \mathrm{R} \mid \mathrm{px}=0\} \text { ( } \mathrm{R} \text { has no nilpotent elements) }
$$

Let $a \neq 0$ be an element of $R$.

Case 1. a $\varepsilon \mathrm{T}(\mathrm{R}) \quad \mathrm{a}=\mathrm{a}_{1}+\mathrm{a}_{2}+\ldots+\mathrm{a}_{t}, \mathrm{a}_{1} \varepsilon \mathrm{R}_{\mathrm{P}_{1}}$

If $\bar{a}=\overline{0}$, we are done. Otherwise $\bar{a} \neq \overline{0}$ and by (ii) there exists an integer $s$ such that $1 \leq s \leq t$ with $a_{s} \varepsilon R_{p_{s}}=R_{p}$ and $\bar{a}=\bar{a}_{s}$ in $A$. But for some collection of integers $m_{1}, m_{2}, \ldots, m_{k}, m_{k}\left(a_{s}\right)^{k}+\ldots+m_{2}\left(a_{s}\right)^{2}+m_{1} a_{s}=0$ and $\mathrm{m}_{1} \mathrm{a}_{\mathrm{s}} \neq 0$. This implies that $\mathrm{p}$ does not divide $\mathrm{m}_{1}$ and

$$
\begin{aligned}
\overline{0} & =m_{k}\left(\bar{a}_{s}\right)^{k}+m_{k-1}\left(\bar{a}_{s}\right)^{k-1}+\ldots+m_{2}\left(\bar{a}_{s}\right)^{2}+m_{1} \bar{a}_{s} \\
& =m_{k}(\bar{a})^{k}+m_{k-1}(\bar{a})^{k-1}+\ldots+m_{2}(\bar{a})^{2}+m_{1} \bar{a} .
\end{aligned}
$$

Since $\bar{a}=\bar{a}_{s} \neq \overline{0}$ we also have $m_{1} \bar{a}=m_{1} \bar{a}_{s} \neq \overline{0} \quad\left(p\right.$ does not divide $m_{1}$ ).

Hence $\bar{a}$ is QAI.

Case 2. a $\neq \mathrm{T}(\mathrm{R})$

If $\bar{a}=\overline{0}$ in $A$, we are done. Suppose that $\bar{a} \neq \overline{0}$. Since $R$ is a QAI-ring, there exist integers $n, r_{1}, r_{2}, \ldots, r_{n}$ such that $r_{n} a^{n}+r_{n-1} a^{n-1}+\ldots+r_{2} a^{2}+r_{1} a=0$ with $r_{1} a \neq 0$.

If $p$ does not divide $r_{1}$, then $r_{1} \bar{a} \neq \overline{0}$ in $A$ and we are finished. 
If $p$ divides $r_{1}$, then $\overline{0}=r_{n}^{-\frac{n}{a}}+r_{n-1} \bar{a}^{-n-1}+\ldots+r_{2} \bar{a}^{2}+r_{1} \bar{a}^{-}$

$$
\begin{aligned}
& =r_{n^{a}}^{-n}+r_{n-1} \bar{a}^{-n-1}+\ldots+r_{2^{a}}^{-2} \\
& =\left(r_{n}^{-n-1}+r_{n-1} \bar{a}^{-n-2}+\ldots+r_{2}^{-a}\right) \bar{a}
\end{aligned}
$$

But $A$ has no zero divisors and $\bar{a} \neq \overline{0}$ so $r_{n^{-}}^{-n-1}+\ldots+r_{3^{-a}}{ }^{-2} r_{2} \bar{a}=\overline{0}$.

If $p$ does not divide $r_{2}$, then $r_{2} \bar{a} \neq \overline{0}$ in $A$ and we are finished. If $p$ divides $r_{2}$, then as above we have $r_{n}(\bar{a})^{n-2}+\ldots+r_{3} \bar{a}=\overline{0}$. Continuing in this manner, it follows that either $\bar{a}$ is QAI by (i) or p divides $r_{i}$ for $1=1,2, \ldots, n$.

Therefore, assume $p$ divides $r_{i}$ for $i=1,2, \ldots, n$, and suppose $p^{t}$ is the highest power of $p$ which divides all of the $r_{i}$. Write $r_{j}=p^{t} b_{j}$ for some integers $b_{1}, b_{2}, \ldots, b_{n}$; at least one of these integers, say $b_{y}$, is not divisible by $p$.

Now, $0=r_{n} a^{n}+\ldots+r_{2} a^{2}+r_{1} a=p^{t}\left(b_{n} a^{n}+\ldots+b_{2} a^{2}+b_{1} a\right)$.

Set $z=b_{n} a^{n}+\ldots+b_{2} a^{2}+b_{1} a$. If $z=0$ in $R$, then $\bar{a}$ is QAI by 1 , since $\left(b_{y}, p\right)=1$. Otherwise $z \neq 0$ and since $p^{t} z=0, z \in T(R)$; in fact, $z \in R_{p}$.

By Case 1 , either $\bar{z}=\overline{0}$ in A which implies $b_{n} \bar{a}^{-n}+\ldots+b_{2} \bar{a}^{2}+b_{1} \bar{a}=\overline{0}$ and we are finished again by 1 ; or $\bar{z} \neq \overline{0}$ in $A$. If this is the case, (R is QAI) then for some integers $u, c_{1}, c_{2}, \ldots, c_{u} ; c_{1} z \neq 0$ and $c_{u} z^{u}+\ldots+c_{2} z^{2}+c_{1} z=0$. $c_{1} z \neq 0$ implies $p$ does not divide $c_{1}$. Then $\overline{0}=c_{u}(\bar{z})^{u}+\ldots+c_{2}(\bar{z})^{2}+c_{1} \bar{z}$ and $c_{1} \bar{z} \neq \overline{0}$ in $A$. This gives $\begin{aligned} \overline{0}=c_{u}(\bar{z})^{u} & +\ldots+c_{2}(\bar{z})^{2}+c_{1}(\bar{z})=c_{u}\left(b_{n}^{-a^{n}}+\ldots+b_{2} \bar{a}^{2}+b_{1} \bar{a}\right)^{u}+\ldots \\ & +c_{2}\left(b_{n} \bar{n}^{-n}+\ldots+b_{2} \bar{a}^{-2}+b_{1} \bar{a}\right)^{2}+c_{1}\left(b_{n}-n+\ldots+b_{2}^{-a^{a}}+b_{1} \bar{a}\right) .\end{aligned}$ 
Note that the coefficient of $\bar{a}$ in the last equation is $c_{1} b_{1}$. If $p$ does not divide $b_{1}$, then $p$ does not divide $c_{1} b_{1}$ which implies $\bar{a}$ is QAI by (I).

If $p$ divides $b_{1}$ but does not divide $b_{2}$, let

$w=z-b_{1} a=b_{n} a^{n}+\ldots+b_{3} a^{3}+b_{2} a^{2}$. Then $\bar{w}=\overline{z-b_{1} a}=\bar{z}-b_{1} \bar{a}=\bar{z}$,

because $A$ has characteristic $p$, which divides $b_{1}$.

$\overline{0}=c_{u}(\bar{w})^{u}+\ldots+c_{2}(\bar{w})^{2}+c_{1} \bar{w}=c_{u}\left(b_{n^{a}} \bar{a}^{n}+\ldots+b_{2} \bar{a}^{2}\right)^{u}+\ldots$

$\left.+c_{2}\left(b_{n}^{-\frac{n}{a}}\right)+\ldots+b_{2} \bar{a}^{2}\right)^{2}+c_{1}\left(b_{n}^{-n}+\ldots+b_{2} a^{-2}\right)$.

Note that the coefficient of $\bar{a}^{2}$ here is $c_{1} b_{2}$, and since $p$ does not divide $c_{1} b_{2}, \bar{a}$ is QAI by ( 1 ). If $p$ divides $b_{1}$ and $b_{2}$ but not $b_{3}$, let $v=z-b_{1} a-b_{2} a^{2}$ and do as before, since again $\bar{v}=\bar{z}$. Since there is $b_{y}$ which is not divisible by $p$ we eventually reach a stage where $\bar{a}$ is QAI by (i). In any case, $a$ in $R$ implies $\bar{a}$ is $Q A I$. Hence $A=R / c(M)$ is a $Q A I-r i n g$.

THEOREM 32. Suppose $R$ is a QAI-ring. Then $R$ is a subdirect sum of prime QAI-rings and hence a subdirect sum of periodic fields and orders in algebraic rational division algebras.

PROOF. Let $F$ be the family of all maximal multiplicative systems in $R$. By Lemma $30, R$ is a subdirect sum of the integral domains $\{R / c(M)\}_{M \in F}$, and Lemma 31 implies that each $R / c(M)$ is a prime QAI-ring. Theorem 18 and Theorem 19 yield the desired form for the subdirect summands.

The example at the end of Section 2 shows that the converse of Theorem 32 fails to hold. The reader is encouraged to compare Theorem 32 to [5, Theorem 14.7]. 
ACKNOWLEDGMENT. A majority of this paper appeared in the Ph.D. dissertation written under the direction of Professor F. Kosier at the University of Iowa. The author would like to express his appreciation to Professor Kosier for his help and guidance.

\section{REFERENCES}

1. Hentzel, Irvin Roy. Alternative Rings Without Nilpotent Elements, Proc. Amer. Math. Soc. 42 (1974), 373-376.

2. Jacobson, N. Structure Theory for Algebraic Algebras of Bounded Degree, Ann. Math. 46 (1945), 695-707.

3. Jacobson, N. Structure of Rings, American Mathematical Soclety, Providence, R. I., 1964.

4. Luh, Jiang and J. C. K. Wang. The Structure of a Certain Class of Rings, Math. Japon. 20 (1975), 149-157.

5. Osborn, J. Marsha11. Varieties of Algebras, Advances in Math. 8 (1972), 163-369. 


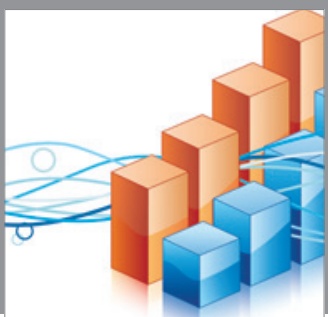

Advances in

Operations Research

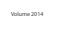

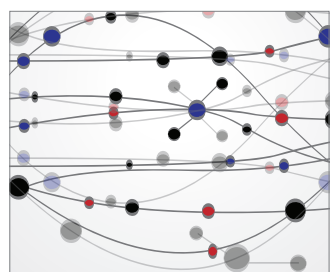

\section{The Scientific} World Journal
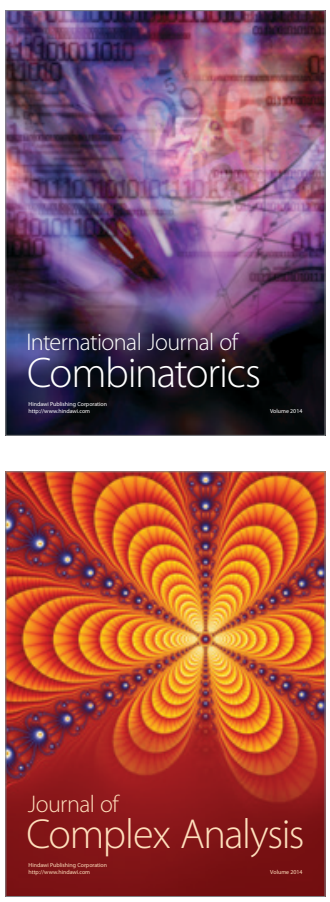

International Journal of

Mathematics and

Mathematical

Sciences
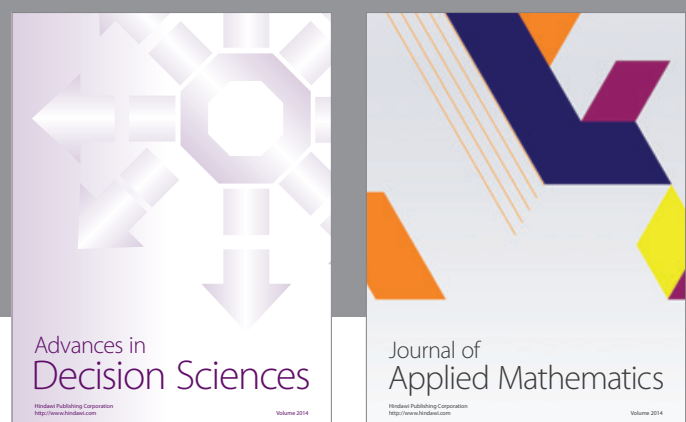

Journal of

Applied Mathematics
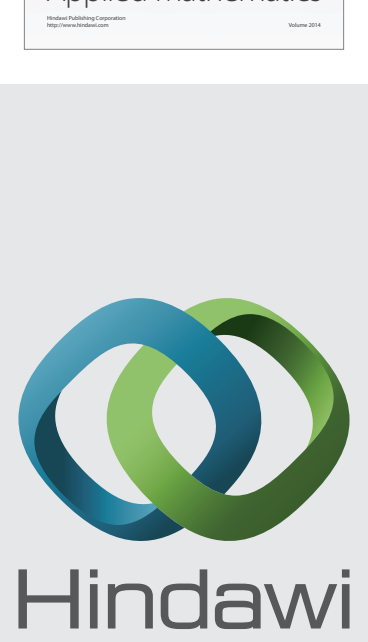

Submit your manuscripts at http://www.hindawi.com
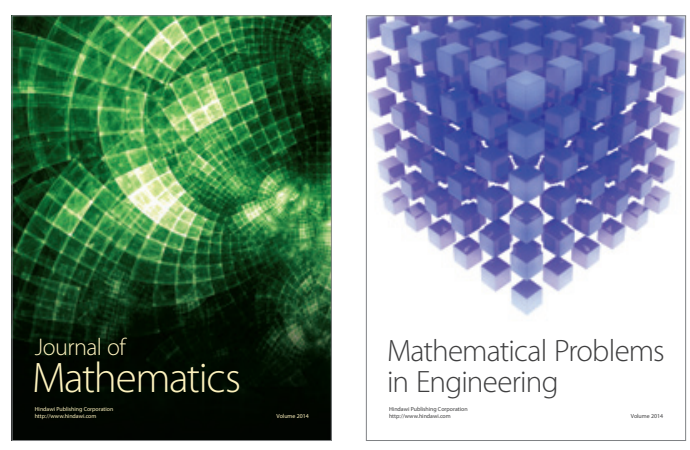

Mathematical Problems in Engineering
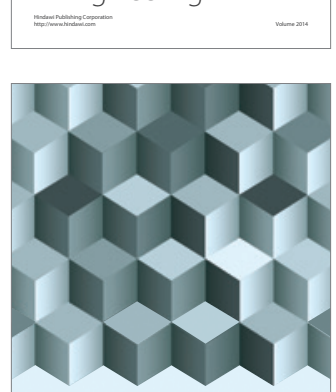

Journal of

Function Spaces
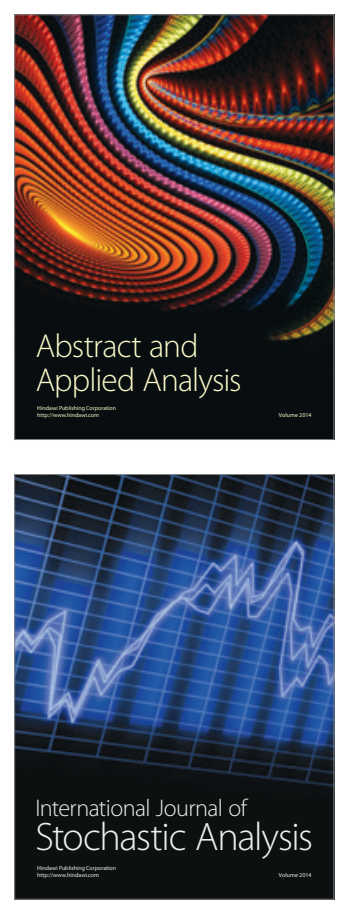

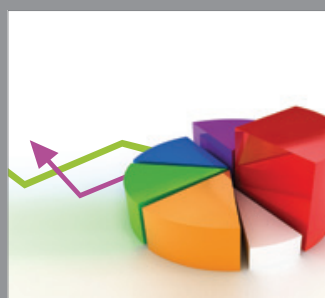

ournal of

Probability and Statistics

Promensencen
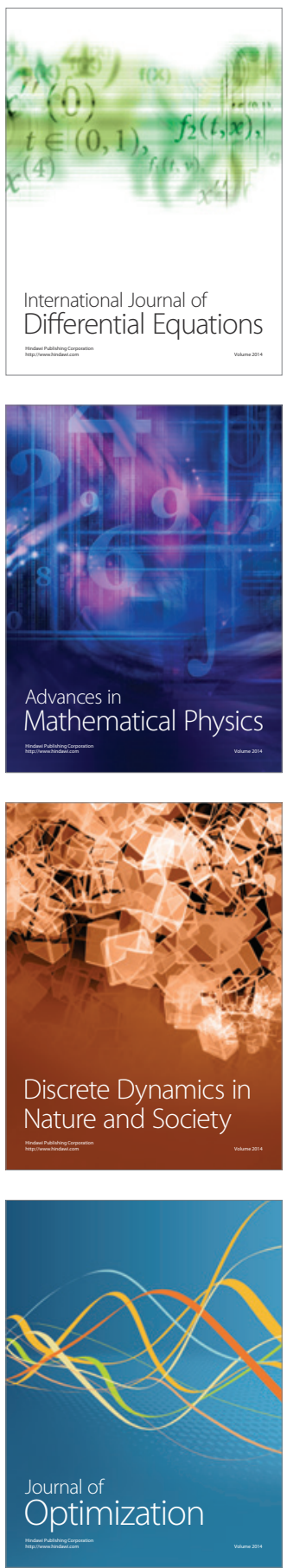